\title{
L'attaque des clowns : emprunts parodiques du cinéma latino-américain aux blockbusters hollywoodiens
}

\section{Emmanuel Vincenot}

\section{(2) OpenEdition}

\section{Journals}

Édition électronique

URL : https://journals.openedition.org/cher/7247

DOI : $10.4000 /$ cher.7247

ISSN : 2803-5992

Éditeur

Presses universitaires de Strasbourg

Édition imprimée

Date de publication : 7 juillet 2014

Pagination : 265-279

ISBN : 978-2-86820-569-8

ISSN : 1968-035X

\section{Référence électronique}

Emmanuel Vincenot, «L'attaque des clowns : emprunts parodiques du cinéma latino-américain aux blockbusters hollywoodiens », reCHERches [En ligne], 12 | 2014, mis en ligne le 13 décembre 2021, consulté le 15 décembre 2021. URL : http://journals.openedition.org/cher/7247 ; DOI : https://doi.org/ 10.4000/cher.7247

\section{(c) (1) (2)}

Ce(tte) œuvre est mise à disposition selon les termes de la Licence Creative Commons Attribution Pas d'Utilisation Commerciale - Partage dans les Mêmes Conditions 4.0 International. 


\title{
L'attaque des clowns : emprunts parodiques du cinéma latino-américain aux blockbusters hollywoodiens
}

\author{
Emmanuel Vincenot
}

Université de Tours

orsque l'on évoque le cinéma latino-américain, c'est souvent une
définition restrictive qui prévaut. De manière récurrente, les études consacrées aux films venus d'Amérique latine se concentrent sur la partie «noble» de la production, à savoir les œuvres s'inscrivant dans une conception auteurisante du cinéma, consacrées par la critique et reconnues pour leur valeur esthétique. Si cette attitude est parfaitement compréhensible, elle présente pourtant l'inconvénient de laisser hors du champ scientifique une abondante production, souvent dominante en termes quantitatifs, qui sait, ou a su, réunir un public nombreux. Or ce cinéma méconnu, que l'on qualifiera par commodité de "populaire», et qui reste dans l'ombre autant pour des questions de préjugés que d'accessibilité des œuvres, offre de fertiles perspectives de recherche et de réflexion, pour peu que l'on évacue la question de la valeur artistique.

Le cinéma "populaire» tel que je l'entends ici s'adresse à un public à la fois vaste et circonscrit (les couches de la société considérées comme inférieures) en lui promettant un moment de divertissement bon marché (que ce soit par le rire, le frisson ou les larmes) le tout dans un but exclusivement commercial (son modèle économique est celui de l'industrie hollywoodienne). Les promoteurs de ce cinéma ne se soucient pas d'être reconnus par des instances évaluatrices "supérieures», telles que la critique ou le monde universitaire. Ils agissent en fait en dehors de leur sphère et de leur portée. Leur production se caractérise par le recours à des formules 
génériques solidement établies, qui peuvent varier en fonction des modes du moment, mais où la comédie est souvent présente. Comme l'a déjà souligné Mikhail Bakhtin (Bakhtin 1970: 11), le rire est depuis l'antiquité une composante essentielle de la culture populaire et le cinéma latino-américain en offre une nouvelle illustration. Dans ce cinéma comique, la farce et le burlesque sont incontournables (on les retrouve par exemple dans les sexycomedias mexicaines, ou les chanchadas brésiliennes) mais d'autres registres peuvent être utilisés, comme la parodie.

Il est ainsi possible d'identifier un corpus de films parodiques latinoaméricains qui multiplient les emprunts aux grands succès du cinéma hollywoodien de ces quarante dernières années pour en proposer, à un public qui connaît parfaitement les œuvres citées, une relecture déformante. Sans prétendre étudier l'intégralité de cette production, je me propose, après avoir cerné les contours de la parodie, d'analyser certaines des réalisations les plus emblématiques du genre afin de mettre à jour leurs mécanismes et leurs discours latents. Je tenterai également de montrer comment les emprunts parodiques mexicains se distinguent de ceux des autres productions latinoaméricaines.

\section{Parodiez, parodiez, il en restera toujours quelque chose}

Depuis Aristote, la parodie a fait l'objet de nombreux débats et sa définition, comme son évaluation, a souvent varié au cours de l'histoire. D'abord limitée au statut de technique citationnelle, la parodie a fini par acquérir le statut de genre littéraire, auquel le cinéma a offert un débouché naturel.

Ainsi que le rappelle Gérard Genette dans Palimpsestes (Genette 1982: 41), la parodie ne doit pas être confondue avec le pastiche: alors que le pastiche relève de l'imitation (d'un style), la parodie est une transformation (d'un texte singulier). En quoi consiste cette transformation? Pour Daniel Sangsue, qui poursuit la réflexion de Genette, elle est ludique, comique ou satirique (Sangsue 1994: 74).

L'intention parodique, pour sa part, est plus difficile à évaluer, et elle suscite des jugements contradictoires: à certaines époques, c'est une conception négative de la parodie qui a prévalu, la transformation de l'hypotexte étant considérée comme une négation de ce dernier. La parodie effectuerait un travail de sape; il s'agirait d'un «acte d'opposition littéraire» (Sangsue 1994: 75). Mais, comme l'ont fait remarquer d'autres théoriciens, la parodisation implique une reconnaissance préalable de la cible, car 
l'hypotexte doit être jugé digne d'être transformé. Le geste parodique serait essentiellement admiratif.

En fait, la parodie est bien souvent paradoxale, voire schizophrène, et c'est là tout son intérêt. Pour Daniel Sangsue, la parodie est un mélange de dépendance et d'indépendance. Elle s'évertue à défaire ce qu'elle vénère.

Logiquement, la parodie s'exerce donc souvent sur des grandes œuvres, sur tout texte qui jouit d'une forme de reconnaissance et de célébrité. L'une des premières parodies de l'Histoire, la Deiliade, reprenait ainsi L'Iliade d'Homère. La Bible a été parodiée un nombre incalculable de fois, de même que les tragédies antiques et classiques, ou les mythes modernes, comme celui de Frankenstein.

Pour ce qui est du cinéma latino-américain, on y retrouve sans surprise la même tendance à reprendre et à transformer les œuvres les plus célèbres: pendant longtemps, l'inspiration a été littéraire, les mythes et les personnages populaires, comme Don Juan, les Trois Mousquetaires ou Pinocchio, ayant inspiré les parodistes du continent; mais depuis les années 1970, ce sont principalement les grands succès du cinéma hollywoodien qui font l'objet d'emprunts parodiques. Ont ainsi été transformés avec plus ou moins de bonheur Jaws (Spielberg, 1975), Star Wars (Lucas, 1977), Indiana Jones (Spielberg, 1981), E.T. (Spielberg, 1982), Rambo (Ted Kotcheff, 1982), Batman (Tim Burton, 1989) ou plus récemment Harry Potter (Chris Columbus, 2001).

Parodies latino-américaines de blockbusters (liste non exhaustive)

\begin{tabular}{|c|c|}
\hline Parodie & Hypotexte \\
\hline $\begin{array}{l}\text { Bacalhau } \\
\text { (Adriano Stuart, 1975) }\end{array}$ & $\begin{array}{l}\text { Jaws } \\
\text { (Steven Spielberg, 1975) }\end{array}$ \\
\hline $\begin{array}{l}\text { Costinha e o King Mong } \\
\text { (Alcino Diniz, 1977) }\end{array}$ & $\begin{array}{l}\text { King Kong } \\
\text { (John Guillermin, 1976) }\end{array}$ \\
\hline $\begin{array}{l}\text { Os Trapalhoes na guerra dos planetas } \\
\text { (Adriano Stuart, 1978) }\end{array}$ & $\begin{array}{l}\text { Star Wars } \\
\text { (George Lucas, 1977) }\end{array}$ \\
\hline $\begin{array}{l}\text { Los extraterrestres } \\
\text { (Enrique Carreras, 1983) }\end{array}$ & $\begin{array}{l}\text { E.T. } \\
\text { (Steven Spielberg, 1982) }\end{array}$ \\
\hline $\begin{array}{l}\text { Etéia, a Extraterrestre em Sua Aventura no Rio } \\
\text { (Roberto Mauro, 1984) }\end{array}$ & $\begin{array}{l}\text { E.T. } \\
\text { (Steven Spielberg, 1982) }\end{array}$ \\
\hline $\begin{array}{l}\text { Rambito y Rambón, primera misión } \\
\text { (Enrique Carreras, 1986) }\end{array}$ & $\begin{array}{l}\text { Rambo, First Blood } \\
\text { (Ted Kotcheff, 1982) }\end{array}$ \\
\hline $\begin{array}{l}\text { Las locuras del extraterrestre } \\
\text { (Carlos Galletini, 1988) }\end{array}$ & $\begin{array}{l}\text { E.T. } \\
\text { (Steven Spielberg, 1982) }\end{array}$ \\
\hline $\begin{array}{l}\text { Tambo } \\
\text { (Antonio Ornelas, 1990) }\end{array}$ & $\begin{array}{l}\text { Rambo, First Blood } \\
\text { (Ted Kotcheff, 1982) }\end{array}$ \\
\hline $\begin{array}{l}\text { Tijuana Jones } \\
\text { (Gilberto de Anda, 1991) }\end{array}$ & $\begin{array}{l}\text { Raiders of the Lost Ark } \\
\text { (Steven Spielberg, 1981) }\end{array}$ \\
\hline $\begin{array}{l}\text { Barman y Droguín } \\
\text { (Gilberto de Anda, 1991) }\end{array}$ & $\begin{array}{l}\text { Batman } \\
\text { (Tim Burton, 1989) }\end{array}$ \\
\hline $\begin{array}{l}\text { Jarry Putter } \\
\text { (Víctor Manuel Castro, 2003) }\end{array}$ & $\begin{array}{l}\text { Harry Potter and the Sorcerer's Stone } \\
\text { (Chris Columbus, 2001) }\end{array}$ \\
\hline
\end{tabular}




\section{Dis-moi qui tu parodies, je te dirai qui tu es}

Avant d'analyser la façon dont s'est effectuée la transformation parodique des hypotextes filmiques recensés dans le tableau ci-dessus, une double question se pose: celle du lieu de production des parodies et celle du choix des cibles.

Concernant le premier point, il convient de signaler que seuls trois pays ont produit des films parodiques: le Brésil, le Mexique et, dans une moindre mesure, l'Argentine. Parmi les titres que nous venons de citer, Bacalhau, Costinha e o King Mong, Os Trapalhoes na guerra dos planetas et Etéia, a Extraterrestre em Sua Aventura no Rio sont brésiliens, Tijuana Jones, Barman y Droguín, Tambo et Harry Putter sont mexicains, tandis que Los extraterrestres, Rambito y Rambón et Las locuras del extraterrestre sont argentins. En revanche, on ne trouve pas trace de parodies en provenance d'autres pays d'Amérique latine, que ce soit par exemple le Pérou, la Colombie ou le Chili, des pays qui possèdent pourtant une tradition cinématographique propre.

En fait, il semble que les cinémas nationaux doivent atteindre une sorte de masse critique pour pouvoir se lancer dans la production de parodies. En Amérique latine, seuls le Brésil, le Mexique et l'Argentine ont réussi à bâtir une industrie cinématographique, d'ailleurs initialement calquée sur le modèle hollywoodien. Seuls ces trois pays ont réussi à développer à certains moments de leur histoire une production suffisamment abondante pour pouvoir concurrencer localement le cinéma états-unien, alors qu'ailleurs sur le continent le cinéma national est resté quantité négligeable. Ces cinématographies mexicaine, brésilienne et argentine sont parvenues à bâtir une tradition reposant sur des genres populaires, des stars mythifiées et des producteurs à succès, une tradition qui a parfois réussi à traverser les frontières. C'est le cas en particulier du cinéma mexicain, qui a conquis un public international dans les années 1940 et 1950 .

La pratique parodique visant essentiellement, comme nous l'avons dit, des films hollywoodiens, il est possible de voir en elle une forme de dialogue avec le modèle originel, avec la matrice des différents cinémas nationaux latinoaméricains ayant connu un développement de type industriel. Dans cette perspective, la parodie serait à la fois le moyen de se poser en interlocuteur et, par conséquent, en égal potentiel du cinéma américain, mais permettrait également de mesurer la différence qui sépare le modèle de ses épigones. Or, depuis une trentaine d'années, la majorité des parodies s'appuient sur un type bien précis de films hollywoodiens: les blockbusters. 
Le blockbuster est un type de produit cinématographique qui apparaît aux Etats-Unis dans les années 1970, et dont le prototype est Jaws. Il s'agit d'un film qui, par rapport aux autres succès que peut produire par ailleurs Hollywood, se distingue par une impressionnante maîtrise technologique, un important effort de marketing et une distribution massive, destinée à écraser la concurrence. De très nombreux films hollywoodiens rencontrent chaque année un succès planétaire, mais tous ne sont pas des blockbusters. Kramer vs. Kramer (Robert Benton, 1979) est ainsi l'un des plus grands succès du box-office des années 1970, mais il ne peut pas être défini comme un blockbuster; il n'entre pas dans la même catégorie de films que Jaws, Star Wars ou Indiana Jones, dont le succès ne repose pas tant sur la capacité à s'inscrire dans une tradition générique (en l'occurrence, celle du mélodrame) que sur les éléments cités précédemment: effets spéciaux époustouflants, lancement du film comme un produit ou comme une marque (les publicitaires parlent de "franchise», ce qui implique l'exploitation de produits dérivés et la réalisation de suites), distribution simultanée sur des milliers d'écrans le même jour (Jaws est le premier titre à avoir introduit ce type de distribution, alors que l'exploitation d'un film s'étalait jusqu'alors sur plusieurs semaines). Le blockbuster, inventé par Steven Spielberg et George Lucas, figures dominantes de ce que Peter Biskind a appelé «le nouvel Hollywood» (Biskind 2002), s'est affirmé depuis les années soixantedix comme le symbole de l'hyperpuissance cinématographique américaine. Depuis sa création, il a permis d'accentuer la présence hollywoodienne sur les écrans du monde entier, et son développement dans les années 1980 et 1990 a coïncidé avec la crise, puis la quasi-disparition de nombreuses cinématographies populaires, principalement dans des pays du Sud. Cela a été le cas en Turquie, en Indonésie, à Hong Kong, mais aussi en Argentine, au Brésil, au Mexique et en Italie.

\section{Hollywood, mon amour}

Dans cette perspective, il est intéressant d'étudier la façon dont les parodistes latino-américains transforment les blockbusters dont ils s'inspirent, comment ils situent leurs œuvres par rapport à ces objets cinématographiques qui incarnent la domination hollywoodienne.

L'une des potentialités de la parodie étant la lecture critique des hypotextes sélectionnés, on pourrait s'attendre à ce que des films tels que Tijuana Jones, Bacalhau ou Rambito y Rambón se moquent de leurs modèles respectifs et, surtout, incitent leurs spectateurs à prendre conscience des 
outils commerciaux, narratifs et psychologiques qu'utilisent les blockbusters, ainsi que du discours qu'ils véhiculent. La parodie pourrait être ainsi le moyen de réfléchir à la vision du monde particulière que développent les films hollywoodiens. Dans le cas de Tijuana Jones, on s'attendrait à ce que la parodie mette à jour l'imaginaire colonial qui habite le film de Spielberg. L'action se déroulant partiellement en Amérique centrale, la parodie pourrait porter sur la représentation négative qui est donnée des LatinoAméricains; elle pourrait, comme le suggère le chercheur brésilien João Luiz Vieira, mettre à jour la portée politique de la démarche d'Indiana Jones, qui parcourt les pays du Sud pour s'emparer de fabuleuses richesses, dans une troublante allégorie du néo-colonialisme (Vieira 1984: 177-178).

Dans les faits, il n'en est rien. Aucun des films que j'ai pu voir ne cherche à réfléchir aux procédés d'écriture filmique et aucun ne propose de lecture que l'on pourrait qualifier de contestataire ou subversive des textes hollywoodiens. De manière surprenante, ces parodies ne débouchent jamais sur un renversement carnavalesque del'hypotexte, sur cette inversion féconde que Mikhail Bakhtin a observée dans la culture populaire du Moyen Age.

C'est particulièrement vrai en ce qui concerne les films argentins Rambito y Rambón et Los extraterrestres. Réalisé en 1986, le premier ne parodie que le titre du film ayant consacré Sylvester Stallone, et se contente de reprendre l'univers militaire de Rambo, sans réutiliser cependant le contexte de la guerre $\mathrm{du}$ Vietnam. Le film repose en fait sur les pitreries de deux acteurs comiques, très populaires à l'époque, Jorge Porcel y Alberto Olmedo, qui incarnent ici deux «troufions» incompétents, provoquant catastrophe sur catastrophe. L'idéologie anti-communiste et l'apologie de la testostérone que propose Rambo n'est absolument pas soulignée par le film argentin, qui s'avère pourtant franchement réactionnaire, sexiste et homophobe (l'armée, qui vient de quitter le pouvoir les mains ensanglantées, est ici présentée comme une institution débonnaire et la caserne ressemble à un aimable camp de vacances; quant aux personnages féminins, ils finissent invariablement en petite tenue). La même idéologie imprègne Los extraterrestres, où un clone d'E.T., qui a atterri en Argentine avec son vaisseau spatial, est poursuivi par des agents secrets communistes (dirigés par un sosie de Lénine), qui veulent mettre E.T. au service de leur cause. Heureusement, Porcel et Olmedo parviennent à renvoyer Monguito (c'est ainsi qu'ils l'ont baptisé) sur sa planète, après avoir utilisé ses pouvoirs pour remporter un combat de boxe et dévêtir plusieurs danseuses de cabaret. 
Dans Tijuana Jones, qui est un croisement d'Indiana Jones I et d'Indiana Jones III, plusieurs séquences se déroulent dans l'univers maya, au fond de la forêt centraméricaine, mais les héros du film, censés être mexicains, n'entretiennent aucune relation de proximité avec la culture maya. Au lieu de questionner la notion d'exotisme, au cœur de la saga de Spielberg, la parodie l'intériorise et la reproduit. C'est particulièrement visible lorsque Tijuana Jones décrypte une inscription en maya à l'entrée d'un temple: l'un de ses compagnons, après lui avoir demandé de parler en "chrétien", s'étonne qu'il sache lire cette langue; Jones lui répond qu'il a simplement lu la traduction en espagnol juste à côté. Le film ne saisit pas l'occasion de rappeler que la culture maya est toujours vivante et que de nombreux citoyens mexicains la perpétuent. Les auteurs du film reprennent par ailleurs les clichés de l'imaginaire exotique américain et font de la forêt du Yucatán un espace de mystère et d'aventures, le lieu d'une altérité radicale. Joao Luis Vieira dresse un constat similaire pour le film O Cangaceiro Trapalhao (Daniel Filho, 1983), qui parodie certaines séquences d'Indiana Jones. Là non plus, le regard dominant que porte le cinéma américain sur le Tiers Monde n'est pas remis en cause (Vieira 1984: 177-178).

Il semble en réalité que la multiplication des parodies de blockbusters ne soit que le symptôme de l'omniprésence des références culturelles états-uniennes dans les sociétés latino-américaines. Cette omniprésence est particulièrement visible dans les productions mexicaines, où de l'intertexte vient se rajouter à la parodie initiale et où chaque film accumule les références à l'univers hollywoodien. Dans Jarry Putter, lors de la cérémonie finale de remise des diplômes de magicien, l'une des élèves porte par exemple une tenue de Catwoman, tandis qu'à un autre moment une des pensionnaires séduit son vieux professeur en lui disant qu'il est le sosie de Brad Pitt (il répond modestement qu'il ne ressemble qu'à Mel Gibson). Le héros de Tambo, homme de ménage dans une salle de sport apparemment réservée aux femmes, vit entouré d'affiches de films d'action mettant en scène Sylvester Stallone et Arnold Schwarzenegger (Rambo, Cobra, Conan le Barbare, Commando). Quant à Tijuana Jones, il met en scène deux sosies des Blues Brothers dans le rôle de tueurs à gages au service d'un méchant nazi.

Le rapport que créent ces parodies de blockbusters avec le cinéma hollywoodien est donc fondamentalement un rapport de soumission. On y distingue la reconnaissance et l'acceptation de la supériorité américaine, source de fascination. La dichotomie inférieur/supérieur est certes intrinsèque au rapport parodique mais il arrive fréquemment qu'elle ne constitue qu'une 
première étape, qui débouche par la suite sur une attitude subversive et critique. Ici, au contraire, la soumission est renforcée, démultipliée par les références au cinéma des USA. Ce que nous disent ces films, c'est que le cinéma, le seul qui compte vraiment, c'est le cinéma hollywoodien.

\section{Made in Ribeirao Preto}

Toute présence du national n'est cependant pas absente des films latinoaméricains, qu'ils soient argentins, brésiliens ou mexicains. On peut même dire qu'il existe, à côté de la tendance que je viens de signaler, une volonté d'ancrer les parodies dans une culture locale (ce qui n'est pas contradictoire, comme j'essaierai de le montrer ultérieurement). Ces films populaires doivent en effet séduire un public local (l'indigence de ces productions leur ferme les portes du marché international) et la connivence culturelle, la mise en partage de références communes sont un élément central de leur stratégie commerciale. Les jeux de mots, les allusions à des gloires nationales, à des personnages historiques, les clins d'oeil à des pratiques sociales, la musique et les chansons: tout cela peut contribuer à donner une identité mexicaine, brésilienne ou argentine aux parodies évoquées. La chanson du générique de Barman y Droguín est ainsi une cumbia (évidemment intitulée la «baticumbia»), un rythme certes d'origine colombienne mais très populaire au Mexique, tandis que Los extraterrestres, filmé dans l'espace d'un cabaret, regorge de numéros chantés, parfois sérieux, mettant en scène des vedettes locales. Dans Rambito y Rambón, les imitations de célébrités se succèdent sans relâche (des soldats s'amusent à contrefaire la voix de commentateurs sportifs, de chanteurs de tango, d'hommes politiques, comme Carlos Menem). Jarry Putter convoque quant à lui la figure du sous-commandant Marcos, cité à plusieurs reprises.

Cette volonté de localiser des hypotextes filmiques à vocation transculturelle se manifeste par ailleurs au niveau générique car, bien souvent, les parodistes semblent chercher à fondre leurs œuvres dans un genre populaire national: la sexy-comedia au Mexique, la pornochanchada au Brésil et l'univers de Porcel et Olmedo (qui est presque un genre à lui tout seul) en Argentine.

Mais ce souci de localisation ne doit pas masquer l'essentiel, à savoir la tendance, plus profonde, à l'autodénigrement. En choisissant de parodier des films qui représentent la forme la plus aboutie de la supériorité technique et financière hollywoodienne, les cinéastes latino-américains 
choisissent délibérément de souligner leur faiblesse économique, leur indigence technique, leur incapacité totale à concurrencer Hollywood. C'est ce qu'affirme Joao Luiz Vieira dans son analyse de deux parodies brésiliennes des années 1970, Bacalhau et Costinha e o King Mong. Comme il le fait remarquer justement, la pathétique réplique du requin des Dents de la mer que l'on aperçoit dans le film porte, lors de sa première véritable apparition, la mention suivante: "Made in Ribeirao Preto" (il s'agit d'une ville de l'État de Sao Paulo). Associer de la sorte la nullité technique de l'effet spécial à son origine géographique (et culturelle) incite le spectateur à se moquer du film lui-même, mais également de la culture et du cinéma brésiliens dans leur ensemble. La différence entre le modèle et sa parodie n'est pas qu'un ressort comique, c'est également un aveu d'impuissance et un acte d'accusation, quasiment mélancolique d'après Vieira (Vieira 1984: 149). Le spectateur ressent le même sentiment d'autodénigrement à la vision d'autres parodies brésiliennes de la même époque, comme Los trapalhoes na guerra dos planetas. Tournée en vidéo, cette adaptation de Star Wars est encore plus indigente techniquement que Bacalhau, le réalisateur n’hésitant pas à montrer des vaisseaux spatiaux fabriqués avec des cuvettes de WC et des sèche-cheveux. La comparaison avec le film de George Lucas est plus que cruelle... Comment, dans ces conditions, le spectateur brésilien, auquel le film est destiné, ne peut-il avoir la certitude de son infériorité, de l'infériorité de son pays et de sa culture?

Si ce discours dépréciatif est globalement absent des parodies argentines (dans les années 1980, lorsque les films furent réalisés, l'Argentine ne se considérait pas et n'était pas considérée comme un pays sous-développé), on le retrouve en revanche dans les films mexicains, avec quelques différences notables.

\section{Mexico is different}

Sil'on prend le cas de Jarry Putter ou de Barman y Droguín, on constate que plusieurs séquences mettent en scène, comme dans les parodies brésiliennes, l'incompétence du responsable des effets spéciaux et du réalisateur, en invitant le spectateur à comparer l'original et la copie. La scène où le vrai Harry Potter reçoit ses premiers cours de pilotage de balai volant est reproduite dans Jarry Putter, mais là où le spectateur attend de magnifiques images de synthèse, il n'a droit qu'à un vieux balai soulevé par deux fils de nylon. Dans Barman y Droguín, le costume des deux héros masqués baille de toutes parts et le principal effet spécial est un gyrophare sur le toit de la 
Batmobile. Là encore, le dénigrement du cinéma national s'accompagne d'une dévalorisation du pays tout entier: lorsque l'un des personnages demande à son ami de lui prêter de l'argent, celui-ci refuse au motif qu'il a plus de dettes que le Mexique lui-même. Et quand il accepte à reculons de se joindre à lui dans sa croisade contre le mal, il fait le commentaire suivant: "Es como salir de Guatemala para meterse en guatepeor». Le nom même que se choisissent les deux protagonistes, Barman et Droguín, sont dévalorisants. Le premier est lié à l'alcool (Barman arbore d'ailleurs non pas la chauve-souris jaune de Batman, mais la chauve-souris blanche et noire qui orne les bouteilles de rhum Bacardi), tandis que le deuxième l'est à la drogue. Mais si ces héros sont de toute évidence des anti-héros, ce n'est pas parce que le parodiste souhaite dévaluer leur modèle hollywoodien, mais tout simplement parce qu'ils sont mexicains et originaires de la capitale, comme le rappelle le surnom ironique qu'ils se sont donné: «el dúo dinámico de chilangolandia».

Pourtant, ce film comporte un deuxième discours, qui s'oppose à celui que je viens d'évoquer et qui propose paradoxalement une défense du cinéma populaire mexicain. Ricardo (Barman dans le couple de justiciers) est en effet fasciné par un film d'action qu'il a vu des dizaines de fois, et il entraîne à plusieurs reprises son acolyte Bruno (Droguín) le voir dans un cinéma de son quartier. Ce film s'intitule Yo el ejecutor (Valentín Trujillo, 1989) et a pour interprète principal Valentín Trujillo, une star du cinéma populaire mexicain des années 1990, qui incarne également le personnage de Ricardo/Barman dans Barman y Droguín. De nombreuses séquences montrent Ricardo et Bruno dans la salle de cinéma, en train de regarder pour la énième fois Yo el ejecutor (on assiste à chaque fois à des scènes de fusillades, de poursuites et d'explosions). L'admiration que voue Ricardo au héros de Yo el ejecutor le pousse à se lancer dans la carrière de justicier masqué et à former avec Bruno le duo de Barman et Droguín. Après avoir remporté leurs premiers succès grâce à leur culot et à leur chance, ils finissent par tomber sur une bande de malfrats bien plus forts qu'eux (c'est la séquence finale). Mais alors qu'on les croit perdus, ils sont sauvés in extremis par... le héros de Yo el ejecutor, qui abat leurs adversaires à coups de fusil à pompe, comme il le fait dans le film dont il s'est échappé. Ricardo / Barman (incarné par Valentín Trujillo) est sauvé par El Ejecutor (également interprété par Valentín Trujillo). L'incarnation de l'incompétence et de l'amateurisme mexicains est ainsi sauvée par un héros du cinéma populaire national. 
Il faut voir dans ce dénouement un plaidoyer (sous forme d'autocélébration cette fois-ci) en faveur d'un cinéma décrié, désigné au Mexique sous le terme de videohome. Apparu entre la fin des années 1980 et le début des années 1990, ce cinéma populaire, distribué exclusivement en vidéo (d'abord en VHS, puis aujourd'hui en DVD) repose sur l'exploitation frénétique de quelques formules à succès (la sexy-comedia, le film de mojados, le film de narcos) et sur un star system uniquement reconnu par le public le moins cultivé1. Ses promoteurs, comme Valentín Trujillo, Gilberto de Anda (le réalisateur de Barman y Droguín) ou les frères Almada (présents de manière intertextuelle dans le film) ont certes conscience de leurs lacunes, mais ils savent aussi que leur cinéma conserve une audience auprès des spectateurs mexicains les plus défavorisés.

Alors que le cinéma populaire brésilien a disparu après les années soixante-dix et que le cinéma populaire argentin n'a pas survécu aux années quatre-vingt, le cinéma populaire mexicain a pour sa part réussi à se maintenir. Difficilement, certes, abaissant progressivement son niveau artistique et technique, renonçant à la pellicule $35 \mathrm{~mm}$, réduisant ses budgets, disparaissant progressivement de la carte culturelle officielle et suivant les migrants dans leur chemin vers les USA, mais cette stratégie a été efficace. Ses productions sont souvent extrêmement violentes et racoleuses, sexistes et machistes, dénuées de toute ambition artistique, mais elles parviennent à offrir aux Mexicains pauvres un cinéma plus accessible que celui distribué dans les multiplexes que fréquente la classe moyenne.

Quand on compare les parodies mexicaines des blockbusters américains aux parodies brésiliennes, on constate que les cinéastes mexicains, tout en étant incapables de proposer une lecture corrosive ou critique des films hollywoodiens, tout en alimentant un sentiment d'infériorité culturelle et en tenant un discours d'autodénigrement qui souligne l'abîme qui sépare l'original de la copie, ne renoncent pas pour autant à imiter sérieusement le cinéma américain, qui les fascine. Que ce soit dans Barman y Droguín, Tambo ou Tijuana Jones, on retrouve la même alternance de moments comiques et de séquences plus sérieuses, où la dynamique parodique est à l'arrêt. Certains passages de Tijuana Jones, à commencer par sa séquence d'introduction, donnent le sentiment que le réalisateur cherche à faire son propre Indiana Jones. Il ne prend pas appui sur un hypotexte pour effectuer

1 Sur les conditions d'apparition du videohome au Mexique, voir Isis Saavedra Luna, Entre la ficción y la realidad. Fin de la industria cinematográfica mexicana, 1989-1994, 2007, Ed. Universidad Autónoma Metropolitana, Mexico. 
une transformation comique, il filme une reprise, un décalque d'Indiana Jones, comme s'il voulait se comparer à l'auteur du film d'origine.

Le spectateur se trouve ainsi face à une copie qui chercherait à se faire passer pour l'original, tout en sachant que le destinataire ne sera pas dupe de la supercherie. Nous sortons du registre exact de la parodie pour entrer sur le terrain du plagiat, même si ce terme ne convient pas vraiment à la situation (ici, la copie n'est pas cachée, elle est au contraire exhibée). Les anglo-saxons possèdent pour leur part un terme péjoratif, "ripoff», qui s'applique beaucoup mieux à ce type de situations textuelles, mais il n'a pas de traduction en français. A défaut d'en proposer une (on peut penser à un mot-valise comme "clonerie» mais il n'est sans doute pas promis à un grand avenir...), je suggère tout simplement d'emprunter à la terminologie juridique et commerciale, et de parler de «film de contrefaçon» ou de «film parasite», de la même façon qu'il existe des marques parasites réalisant ce que la législation française appelle de «l'imitation illicite $»^{2}$.

Il existe en effet une analogie avec l'univers des marques et de la grande consommation: certaines séquences de Tijuana Jones provoquent la même sensation que celle que l'on peut éprouver face à l'imitation évidente d'un produit très connu, comme le Coca-Cola, ou le Nutella. Ces «clones» sont généralement vendus dans les supermarchés à très bas prix (les harddiscounters) et ils s'adressent en priorité à des consommateurs issus des couches populaires, qui n'ont pas les moyens économiques d'accéder aux marques reconnues mais qui sont néanmoins désireux de participer eux aussi à la société de consommation. Tijuana Jones, Jarry Putter ou Tambo entretiennent aux marques qu'ils imitent (j'emploie ce terme à dessein car les blockbusters sont traités par les studios hollywoodiens comme des produits de grande consommation) le même rapport que celui qui existe entre un pot de Pralina acheté chez Leader Price et un authentique pot de Nutella. Les parodies mexicaines proposent à des spectateurs/consommateurs pauvres des versions discount de marques prestigieuses, des copies moins réussies que l'original mais plus accessibles. Tout se passe comme si les cinéastes populaires mexicains, après avoir renoncé à concurrencer frontalement le cinéma hollywoodien, avaient décidé de le faire par le bas, en adoptant une stratégie parasitaire de descente de gamme. Il semble que, jusqu'à présent, cette politique ait été payante puisqu'elle leur a permis de survivre alors que leurs homologues argentins et brésiliens ont, pour leur part, disparu.

2 Lire à ce sujet Michel Toporkoff, Droit de la concurrence déloyale, Ed. Gualino, Paris, 2010, p. 65-104. 


\section{Bibliographie}

Bakhtine M., 1970, L'œuvre de François Rabelais et la culture populaire au Moyen Âge et sous la Renaissance, Paris, Ed. Gallimard.

Biskind P., 2002, Le nouvel Hollywood, Paris, Ed. Le Cherche Midi.

Genette G., 1982, Palimpsestes, la littérature au second degré, Paris, Ed. du Seuil, Coll. Points.

Saavedra Luna, I., 2007, Entre la ficción y la realidad. Fin de la industria cinematográfica mexicana, 1989-1994, Mexico, Ed. Universidad Autónoma Metropolitana. Sangsue D., 1994, La parodie, Paris, Ed. Hachette, 1994.

Toporkoff, M., 2010, Droit de la concurrence déloyale, Paris, Ed. Gualino.

Vieira, J.L., 1984, Hegemony and Resistance, Parody and Carnival in Brazilian Cinema, Ph.D., New York University. 